\title{
Metabolic Presbycusis: Differential Changes in Auditory Brainstem and Otoacoustic Emission Responses with Chronic Furosemide Application in the Gerbil
}

\author{
David M. Mills ${ }^{1}$ and Richard A. Schmiedt ${ }^{2}$ \\ ${ }^{1}$ V. M. Bloedel Hearing Research Center, Department of Otolaryngology, Head E Neck Surgery, University of Washington, \\ Seattle, WA 98195, USA \\ ${ }^{2}$ Department of Otolaryngology, Head $\mathcal{E}$ Neck Surgery, Medical University of South Carolina, Charleston, SC 29425, USA
}

Received: 12 February 2003; Accepted: 3 July 2003; Online publication: 12 November 2003

\section{ABSTRACT}

Auditory characteristics of metabolic or strial presbycusis were investigated using an animal model in which young adult Mongolian gerbils (Meriones unguiculates) were implanted with an osmotic pump supplying furosemide continuously to the round window. This model causes chronic lowering of the endocochlear potential (EP) and results in auditory responses very similar to those seen in quiet-aged gerbils (Schmiedt et al., J. Neurosci. 22:9643-9650, 2002). Auditory function was examined up to one week post-implant by measurement of auditory brainstem responses (ABRs) and distortion product otoacoustic emissions (DPOAEs). Emission "threshold" was defined as the stimulus level required to reach a criterion emission amplitude. Comparing all responses on a "threshold-shift diagram," where emission threshold increases were plotted versus ABR threshold increases, the following results were obtained: (1) On average, the increase of the emission threshold was about $55 \%$ of the increase in ABR threshold, with comparatively little scatter. (2) The main dysfunction in metabolic presbycusis appears to be a decrease in the gain of the cochlear amplifier, combined with an additional, smaller increase in neural threshold, both effects caused by a chronically low EP. (3) For ABR threshold increases over $20 \mathrm{~dB}$,

Correspondence to: David M. Mills, Ph.D. • Box 357923 • University of Washington - Seattle, WA 98195. Telephone: (206) 616-7540; fax: (206) 221-5685; email: dmmills@u.washington.edu the points for the chronic low-EP condition were largely separate from those previously found for permanent acoustic damage. The threshold-shift diagram therefore provides a method for noninvasive differential diagnosis of two common hearing dysfunctions.

Keywords: presbycusis, endocochlear potential, differential diagnosis, distortion product otoacoustic emissions, auditory brainstem response, gerbil

\section{INTRODUCTION}

In the human population, acquired loss of hearing in an individual can be due to any of several underlying conditions. Potential causes include noise damage (e.g., Rosenhall et al. 1990; Lucertini et al. 2002) as well as several types of hearing loss associated with aging, known collectively as presbycusis. Sensory presbycusis is ascribed to the primary loss of outer hair cells (OHCs; e.g., Schuknecht 1964), neural presbycusis to the primary loss of eighth nerve fibers (Schuknecht and Gacek 1993; Felder and SchrottFischer 1995), and metabolic or strial presbycusis to the decline of endocochlear potential (EP; Johnsson and Hawkins 1972; Pauler et al. 1988; Gates et al. 2002). Unfortunately, it is not yet possible to determine unequivocally by noninvasive means the underlying cause of hearing dysfunction, e.g., to uniquely distinguish between $\mathrm{OHC}$ or EP loss as a 
primary cause. It would be useful to have a noninvasive means of making such determinations, in anticipation of the development of specific treatments for different causes of hearing loss.

One approach to such differential diagnosis has been to combine measurements of otoacoustic emissions with pure-tone audiometry, or similar measures of neural threshold useful for experimental animals. This combination of measurements was recently tested in gerbil as to its ability to distinguish between two induced conditions, acoustic damage and acute furosemide intoxication (Mills 2003). Auditory brainstem responses (ABRs) were compared with distortion product otoacoustic emission (DPOAE) measurements, and specific measures were found that allowed the two underlying conditions to be detected at $100 \%$ confidence. This was accomplished by plotting ABR threshold changes versus emission threshold changes measured at corresponding stimulus frequencies. In such threshold-shift plots, essentially complete separation was found for the responses resulting from acoustic damage compared to acute furosemide, at all frequencies where the ABR threshold increase was over $20 \mathrm{~dB}$.

In this earlier study, acoustic damage was applied and the animal was allowed to recover for up to two weeks so that only permanent threshold shift would remain to be measured (e.g., Puel et al. 1998). In addition to its obvious direct application, such an acoustic damage paradigm may also serve as an animal model for sensory presbycusis. In terms of metabolic presbycusis, however, the acute response to systemic furosemide is probably not an adequate model. It is probable that there is additional adaptation of hair cell responses to a persistent low EP, i.e., adaptation or recovery over a period of several days similar to that seen in sound damage. Such adaptations would not have had time to be effective on the scale of hours that was afforded employing the acute response to systemic furosemide application.

As an alternative, the continuous application of furosemide to the round window has been shown to be an excellent model for metabolic presbycusis. Studies in quiet-aged gerbils have shown that there is a hearing loss directly related to EP decline, with little associated hair cell loss (Mills et al. 1990; Schmiedt et al. 1990; Tarnowski et al. 1991; Schulte and Schmiedt 1992; Hellstrom and Schmiedt 1996; Schmiedt 1996; Lang et al. 2003). Detailed agreement was found between auditory responses of these gerbils at three years of age and younger animals implanted with a furosemide pump for a week or more (Schmiedt et al. 2002a). This study strengthens the view that the action of furosemide upon the cochlea is primarily through its effect on the EP (see Mills et al. 1993 and references therein). Most important, this method provides a relatively convenient and accurate animal model of metabolic presbycusis. However, the study did not include sufficiently detailed emission measurements to allow for a direct comparison with the differential diagnosis approach discussed above.

In the present study, mini-osmotic pumps containing furosemide were implanted in young adult Mongolian gerbils, and the animal's auditory function was measured 3-7 days later. Frequency-specific ABR threshold measures were compared with detailed DPOAE measurements, using the same procedures as in the acute furosemide study (Mills 2003). Construction of threshold-shift diagrams confirms that an appropriate combination of emission and audiometric thresholds can be used for differential diagnosis. That is, the effects of sound damage, or sensory presbycusis, can be distinguished noninvasively from that of chronic low-EP conditions characteristic of metabolic presbycusis.

\section{METHODS}

Young adult Mongolian gerbils (Meriones unguiculates), weighing 45-65 g, were obtained from Charles River Laboratories (Wilmington, MA). All procedures were approved by the Institutional Animal Care and Use Committee at the University of Washington. For all animals in the main experimental group, furosemide was infused into the round window niche continuously for 3-7 days using an implanted miniosmotic pump (Alzet Model 2004, Durect Corp., Cupertino, CA). The pumps contained furosemide (American Regent Labs, Shirley, NY) at either 5 or 10 $\mathrm{mg} / \mathrm{ml}$, delivered at the nominal rate of $0.25 \mu \mathrm{l} / \mathrm{h}$.

Procedures for implanting pumps and attached cannulas were similar to those previously reported (Schmiedt et al. 2002a, b). Briefly, gerbils were anesthetized using an intraperitoneal injection of $3.5 \mathrm{mg}$ of pentobarbitol (Nembutal, Abbott). They were also given $0.05 \mathrm{mg}$ of atropine subcutaneously and sterile ophthalmic ointment applied to each eye. Two incisions were made, one on the animal's back for insertion of the pump and the other over the inferior mastoid bulla. Tissue was removed to expose the bulla. The cannula from the pump was fed under the skin to the bulla, where it was passed into the bulla through a series of small holes serving as stress relief. Using an operating microscope, the end of the cannula was carefully placed in the round window niche. The bulla openings were then covered using Durelon carboxylate dental cement and both incisions were closed. The animal was allowed to recover, then housed separately but given normal access to food and water. None of the animals showed signs of pain or infection during recovery. 

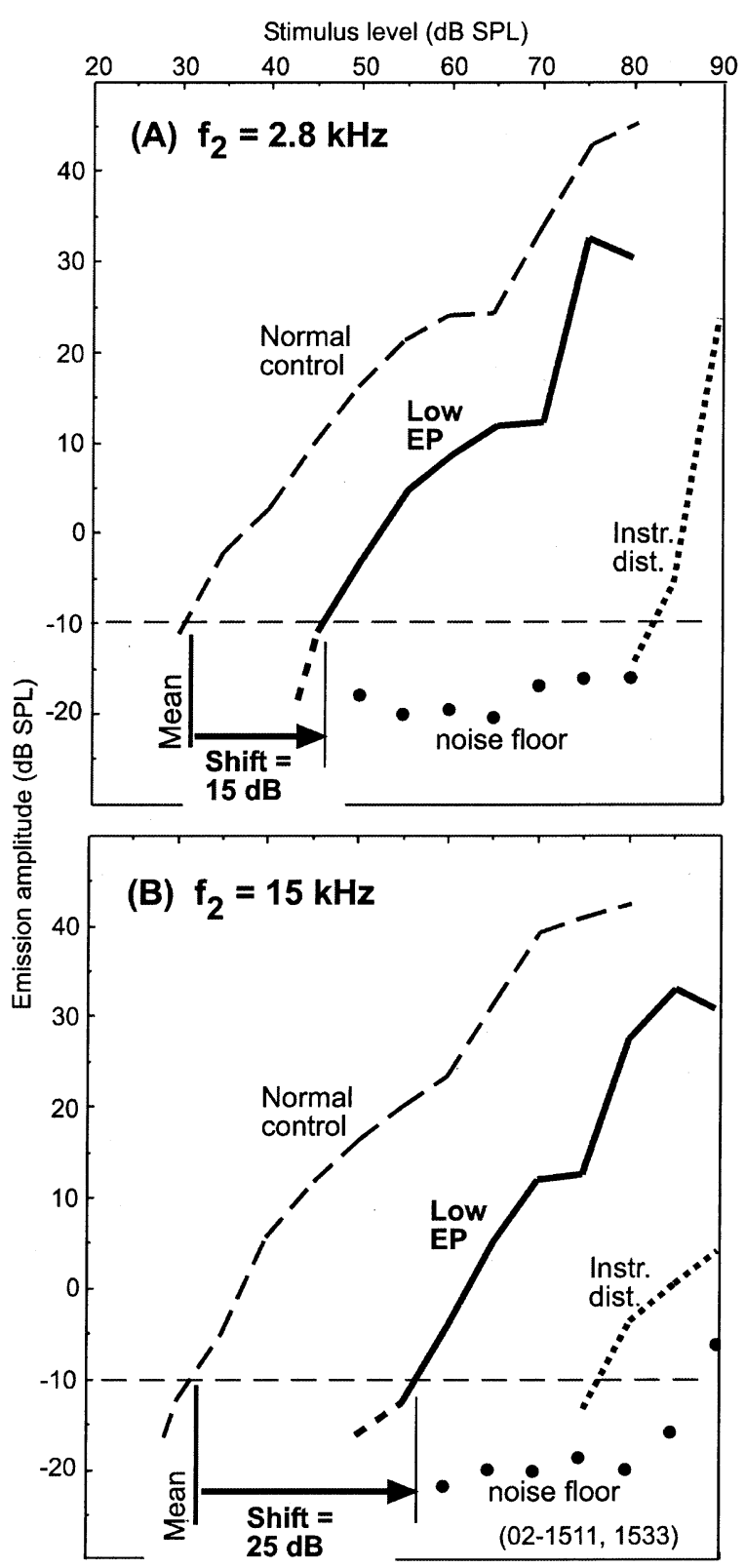

FIG. 1. Typical emission growth functions. Emission amplitude on vertical axis is plotted versus stimulus level on horizontal axis (values indicated at top) at the $f_{2}$ frequencies noted in $\mathbf{A}$ and $\mathbf{B}$. Responses are shown for an experimental animal (bold lines) which had a pump with a concentration of $5 \mathrm{mg} / \mathrm{ml}$ furosemide for 7 days. The response is compared with a typical normal control (dashed lines) as well as with the mean threshold of the normal control group (vertical lines marked "Mean"). The emission threshold is here defined as the stimulus level to obtain an emission amplitude of $-10 \mathrm{~dB}$ SPL (horizontal light dashed lines). Also indicated are estimates of instrumental distortion level (dotted lines at right) and the noise floor (dots) as described in Methods. When emissions in the experimental animal were relatively weak, as in $\mathbf{B}$, maximum stimulus levels were typically increased by $10 \mathrm{~dB}$.

Procedures for measurements of evoked responses were similar to those previously reported (Mills and Shepherd 2001; Mills 2003). Briefly, gerbils were ini- tially anesthetized with a subcutaneous injection of a mixture of ketamine (Ketaject, Phoenix Pharm., 80 $\mathrm{mg} / \mathrm{kg}$ ) and xylazine (Xyla-ject, Phoenix Pharm., 5 $\mathrm{mg} / \mathrm{kg}$ ). Supplemental anesthesia was administered as needed. Typically, the same ketamine-xylazine mixture was given at about $1 \mathrm{~h}$ intervals, plus ketamine alone at the intervening half-hours, at dosages $1 / 4$ to $1 / 2$ the initial injection. The animal was attached to an adjustable surgical head holder (Kopf) with a bite bar and positioned on a heating pad. Animal temperature was maintained at $37^{\circ} \mathrm{C}$. The pinna was removed, as was a small amount of tissue over the superior mastoid bulla cavity. A small hole was drilled into the bulla and a tube $(0.8 \mathrm{~mm}$ ID by 11 $\mathrm{cm}$ long) was force-fit into the hole. The purpose of the small-diameter tube was to provide static pressure relief for the middle ear while maintaining normal middle ear function.

A custom coupler was next advanced under micromanipulator control and sealed to the ear canal. This coupler included a port for a low-noise microphone (Etymotics ER10B), a port for a probe tube reference microphone, and two ports for sound delivered through tubing from custom tweeters. Before beginning measurements, a wide-band noise signal was generated by one tweeter, and the output of the low-noise microphone was calibrated in situ by comparison with the output of the probe tube microphone. The same coupler and calibration were used for both DPOAE and ABR measurements.

For all animals, emission measurements were made first. Stimuli were two tones at frequencies $f_{1}$ and $f_{2}$, with $f_{2} / f_{1}=1.21$. Baseline measurements included input-output, or "growth" functions for both the equal-level stimulus case, $L_{1}=L_{2}$, and the unequal case with $L_{1}=L_{2}+20 \mathrm{~dB}$. Growth functions were measured at approximately half-octave intervals for $f_{2}$ frequencies from 1 to $28 \mathrm{kHz}$. The higher frequencies were shifted from exact half-octave intervals to take advantage of bands of relatively good signal to noise in the microphone characteristic at the emission frequency, $2 f_{1}-f_{2}$. All growth functions were measured with $5 \mathrm{~dB}$ steps in stimulus levels, for stimulus levels chosen for emissions below detectability, up to stimulus levels of $\left(L_{1}, L_{2}\right)$ equal to either $(80,80)$ or $(90,70)$ dB SPL. For every measurement, noise levels were estimated by an appropriate sum of the bin counts at frequencies near $2 f_{1}-f_{2}$ and automatically recorded. Measurements affected by noise were not used to determine threshold values. Examples of measured growth functions are shown in Figure 1, with illustrations of the calculation of emission threshold shifts.

Following emission measurements, ABR responses to tone pips were recorded. Tone pips were generated with a $3 \mathrm{~ms}$ total duration, with $1 \mathrm{~ms} \cos ^{2}$ rise/fall 
(A)
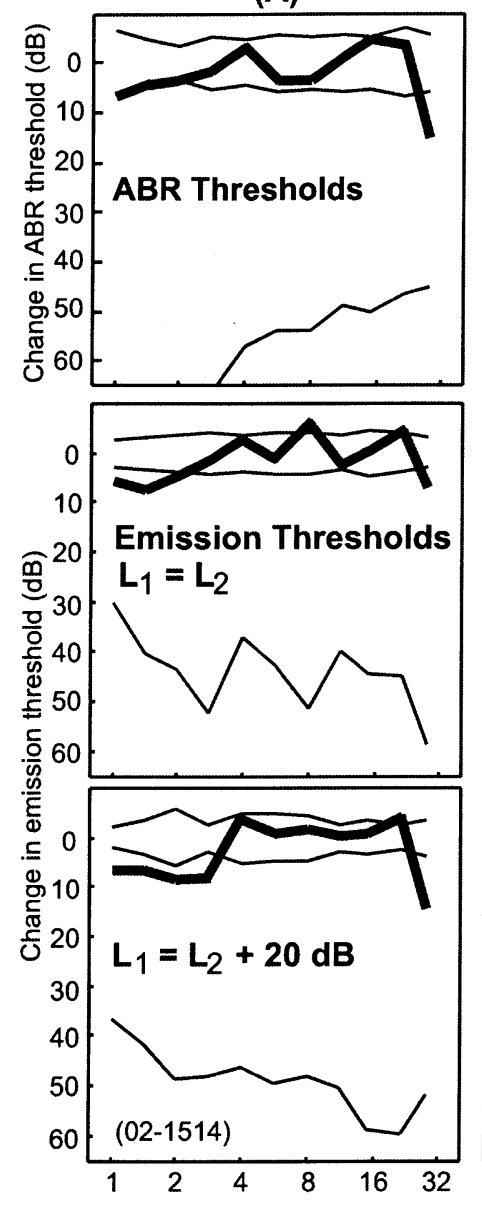

(B)
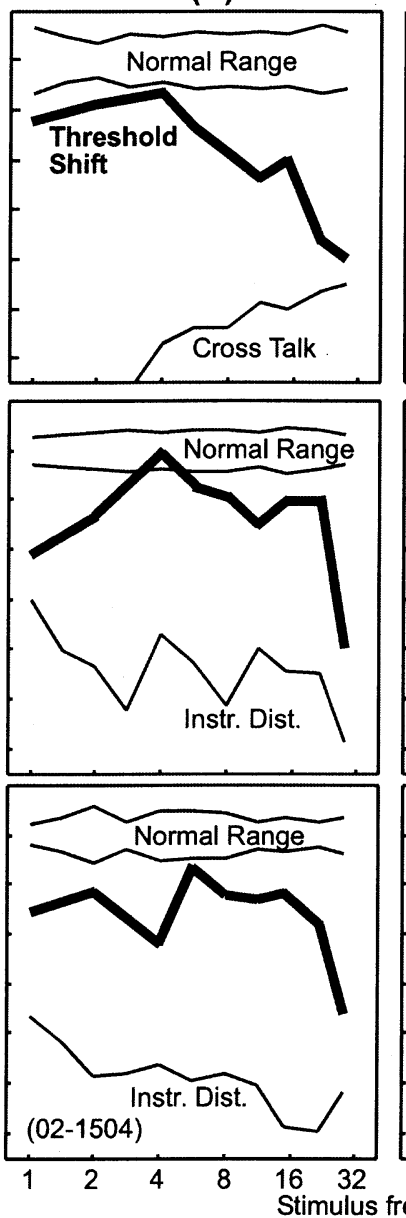

FIG. 2. Range of "audiograms" for animals in the experimental group. Each column (A-D) represents a summary of data for one animal. The top panel in each column represents ABR threshold shifts, the middle panel shows emission threshold shifts for $L_{1}=L_{2}$, and the lower panel illustrates emission threshold shifts for the case $L_{1}=L_{2}+20 \mathrm{~dB}$. Normal ABR and emission thresholds were first determined for a control group of eight gerbils. The normal means were then subtracted from the measured thresholds in the experimental animals to obtain the threshold shifts shown. Note that a
(C)
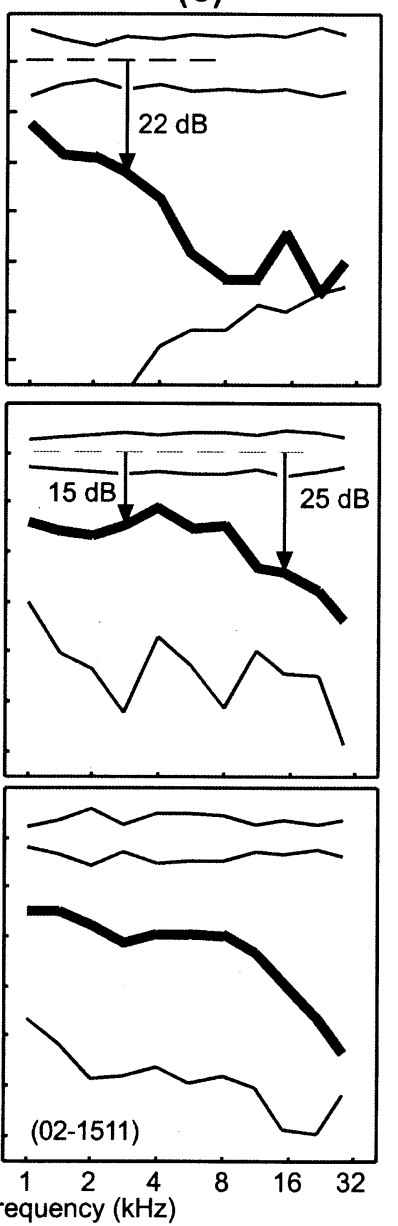

(D)
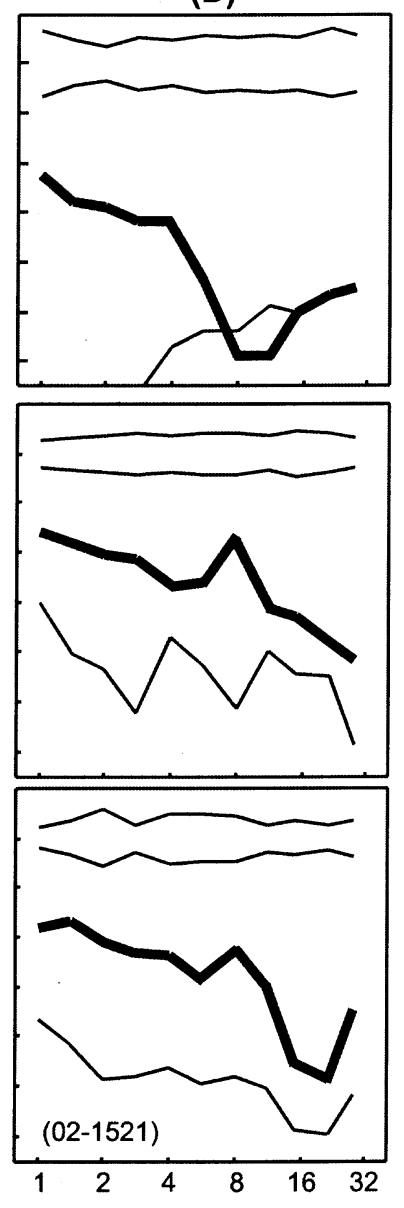

greater increase in threshold is plotted downward in all panels. The normal ranges indicated by the two thin lines at the top of each panel represent the normal means plus and minus one standard deviation. The thin lines at the bottom of the ABR panels represent the estimated threshold for cross talk, i.e., the limitation to the measured threshold elevation for the experimental ear which is due to stimulation of the contralateral ear. Similar lines in the emission audiograms represent estimates of intrinsic instrumental distortion (see Methods).

7 days later (mean 5.8 days). In addition, 8 control animals were measured to determine normal thresholds using the same emission and ABR measurement procedures described above. These were completely normal animals except that a bulla vent tube was installed as described above. Therefore, differences ascribed to the experimental animals include not only the effect of furosemide on the cochlea, but possible differences due to having had additional surgery, effect of the cannula on the middle ear, etc. That such additional effects were small is shown by responses in a number of animals in which the installation of the furosemide pump had little or no detectable effect compared with the control group (e.g., see points clustered around the origin in Figure 4). 


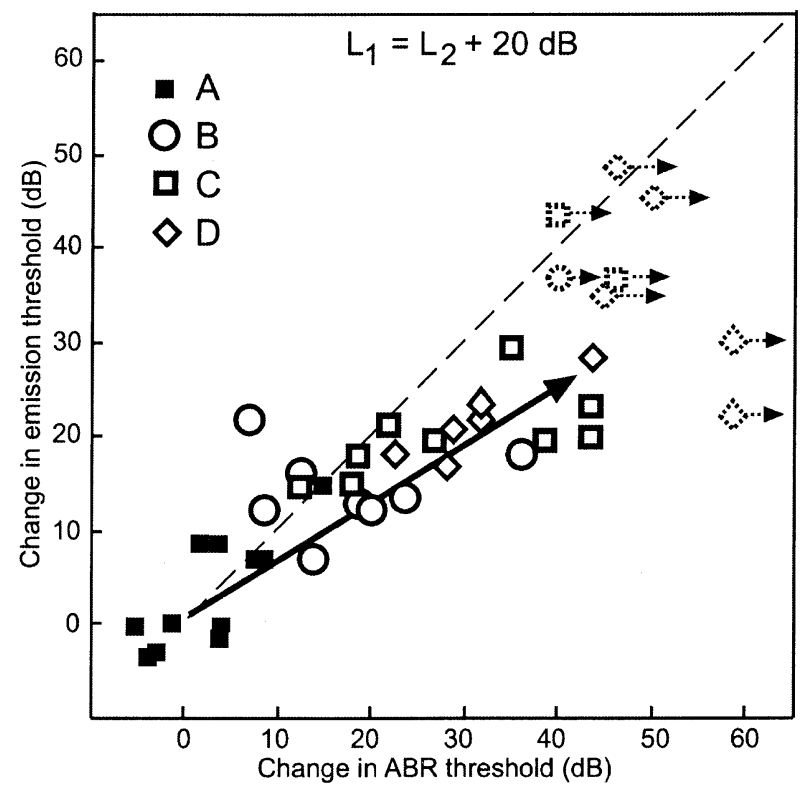

FIG. 3. Threshold-shift diagram for the four animals in Figure 2. The shifts in ABR threshold are shown on the horizontal axis plotted against the shift in emission threshold on the vertical axis. The $45^{\circ}$ dashed line indicates equality in threshold shifts, for reference. The bold arrow indicates the general trend of the shifts with increasing dysfunction. Dashed symbols with arrows represent $A B R$ responses possibly compromised by interaural cross talk.

Finally, two additional types of controls were measured. Instrumental distortion levels for emission measurements were estimated by replacing the gerbil with a long tube with inside diameter approximately equal to the gerbil ear canal (Mills 2002, 2003). The instrumental response essentially limits the amount of emission threshold elevation that can be determined, except as a lower limit. Similarly, measurements of ABR threshold elevations in the experimental group were also limited, but in this case by crosstalk to the contralateral, normal ear. To obtain an estimate of this effect, in several normal animals the ipsilateral cochlea was first destroyed by mechanical disruption through the round window. The round window was then plugged and the bulla hole closed except for a vent tube as described above. ABR measurements were then performed as for the experimental animals.

\section{RESULTS}

Figure 1 displays typical emission input-output, or "growth," functions. The upper panel is for the stimulus frequency, $f_{2}=2.8 \mathrm{kHz}$, the lower is for $f_{2}=15 \mathrm{kHz}$, and both panels are for the case $L_{1}=L_{2}$. In both panels, the emission amplitude measured in the experimental animal is indicated by the heavy

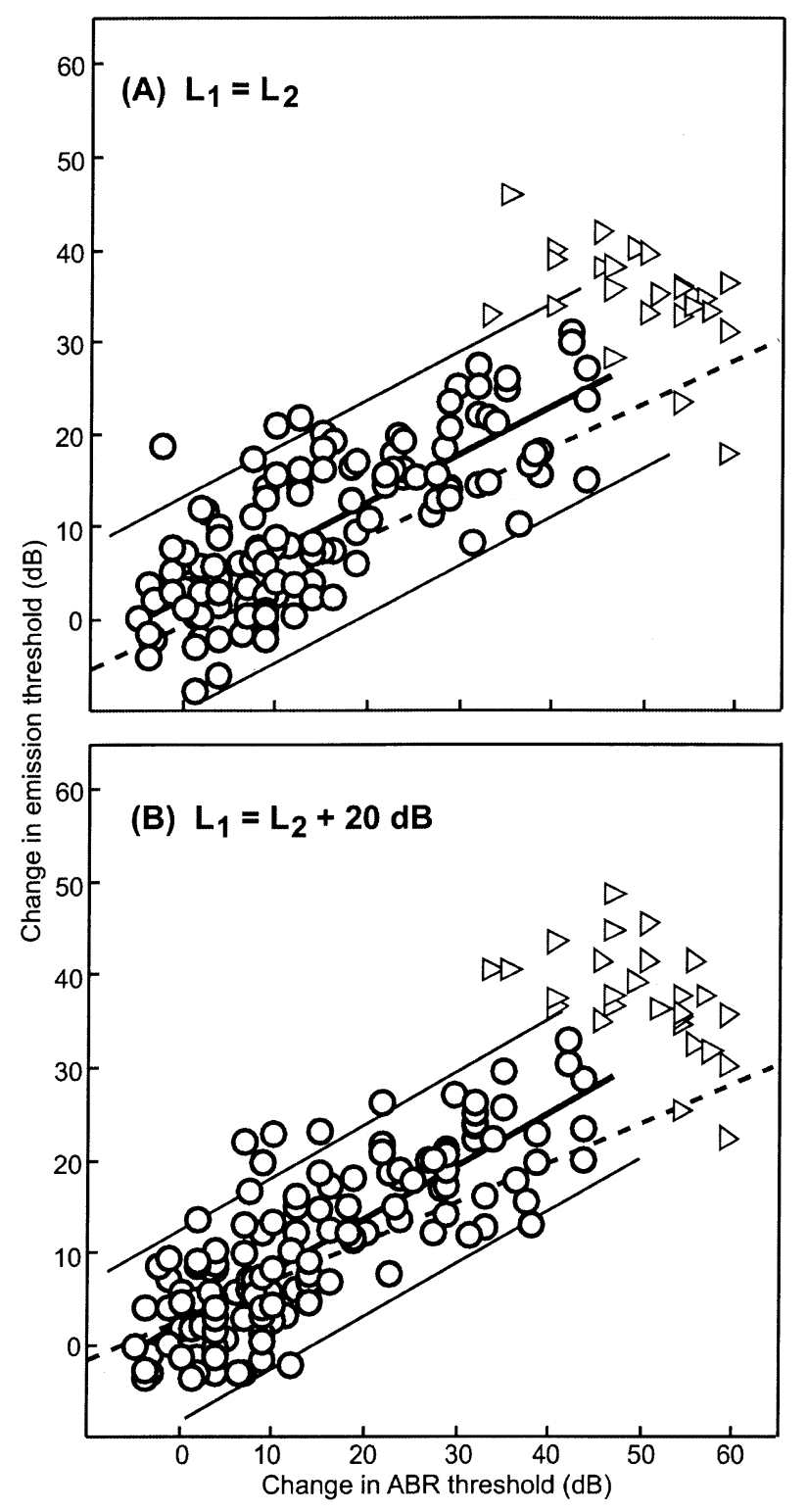

FIG. 4. Threshold-shift diagrams for all animals in the chronic lowEP study. Circles indicate experimental data in which thresholds for $A B R$ and emissions were both below limits set by $A B R$ cross talk and instrumental distortion, respectively (see Fig. 2). Right-facing triangles indicate measurements for which only the lower limit in $A B R$ threshold could be determined because of ABR cross talk. There were no cases in which emission thresholds increased to estimated instrumental distortion levels. In each panel, the heaviest line indicates the least-squares linear fit to the data. For $L_{1}=L_{2}(\mathrm{~A})$, the slope of the regression line is 0.51 and $r^{2}=0.59$, while for $L_{1}=L_{2}+20 \mathrm{~dB}$ (B), the slope is 0.56 and $r^{2}=0.63$. In both cases the regression lines approximately pass through the origin (within $2 \mathrm{~dB}$ ). The pair of lighter solid lines in each panel indicates 95 prediction intervals. The dashed line in each panel shows for comparison the regression line that was previously determined for acute furosemide intoxication (Mills 2003) using the same emission parameters.

solid line. Each panel illustrates a typical calculation of the emission "threshold," defined by the stimulus level required to reach a criterion emission amplitude. 

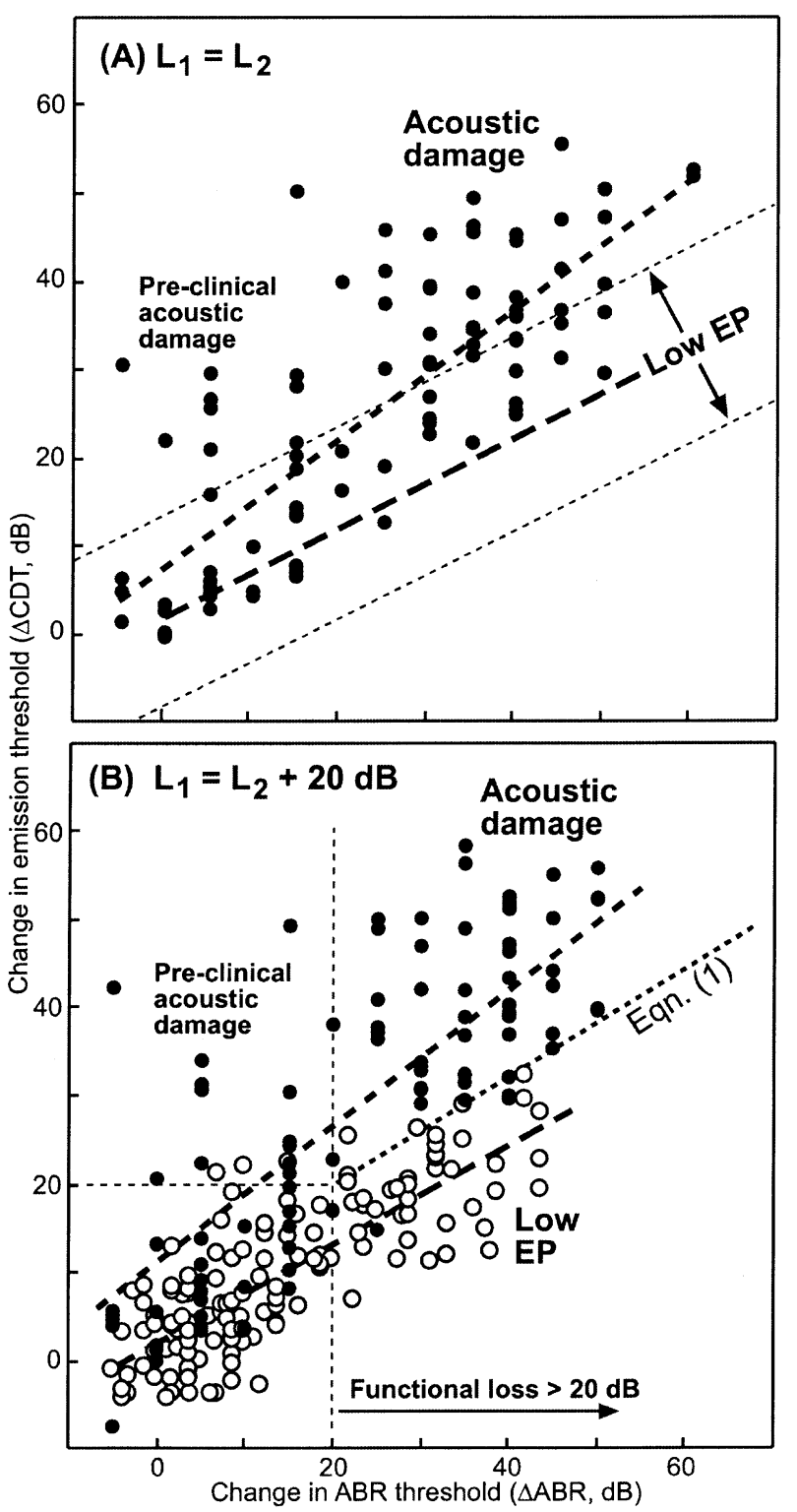

FIG. 5. Threshold-shift diagram comparing the present results with chronic low EP to the previous results for permanent threshold shift caused by acoustic damage (filled circles; Mills 2003). In both panels, the short-dashed bold line indicates the linear regression for the acoustic damage case and the long-dashed bold line indicates the regression line for the chronic low-EP case. A For the equal-level case, there was a lot of overlap between the two dysfunctions. Therefore, to avoid confusion, the approximate extent of the low-EP data is simply indicated by the sloped parallel lines, the 95 prediction intervals from Figure 4. B There was less overlap between the two conditions in the unequal-level case, so symbols are shown for both the chronic low-EP condition (open circles) and the acoustic damage condition (filled circles). The heavy dotted line separating the two distributions is that given by Eq. (1). This line starts at the point where both emission and $A B R$ threshold shifts are equal to 20 $d B$, i.e., at the intersection of the two light-dashed perpendicular lines.

For this report, the criterion level is taken equal to $-10 \mathrm{~dB}$ SPL (horizontal dashed line). Each panel also shows for comparison an example of a growth func-

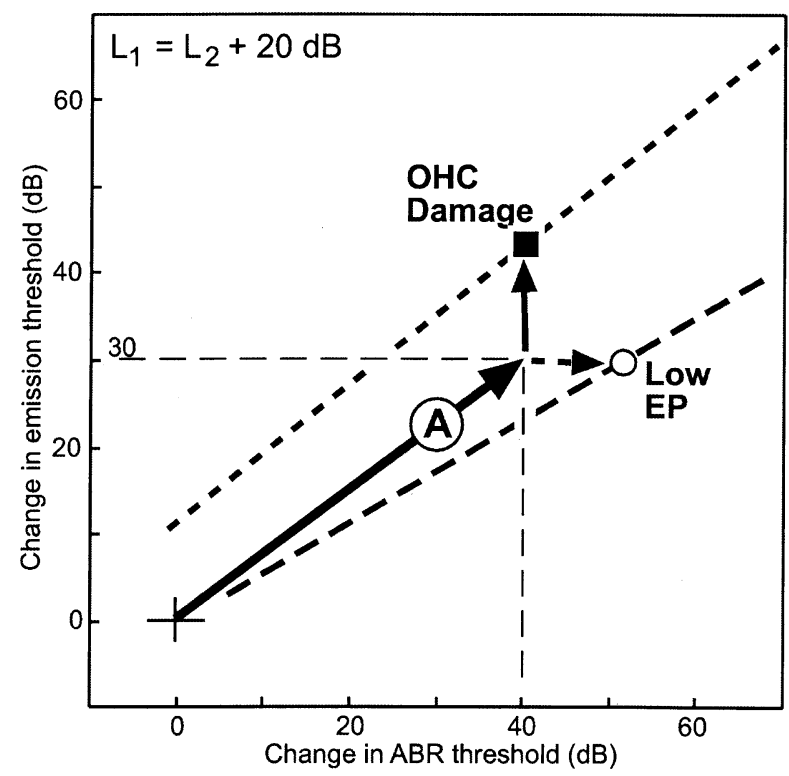

FIG. 6. This diagram shows how changes in cochlear mechanics with different dysfunctions can lead to observed threshold-shift distributions. The dashed lines represent the data, and are the same regression lines shown in Figures $4 \mathrm{~B}$ and $5 \mathrm{~B}$. This diagram represents a thought experiment in which two cases are chosen, one with acoustic damage, the other with a chronic low EP, both of which happen to result in the same decrease in cochlear amplifier gain. The effect of the change in cochlear amplifier gain, taken by itself, results in the vector $\mathbf{A}$, by assumption the same for both dysfunctions. Numerical results were estimated as follows. If the effective cochlear amplifier gain is defined as the amount of increase of the basilar membrane response over the passive case at neural threshold (e.g., Mills 1997, 2003), the gain in $\mathrm{dB}$ is directly related to the associated improvement in neural threshold level. The arrow marked $\mathbf{A}$ is an example of a $40 \mathrm{~dB}$ decrease in effective cochlear amplifier gain, resulting by itself in an increase of $A B R$ threshold equal to $40 \mathrm{~dB}$, noted by the vertical dashed line. This decrease in cochlear amplifier gain will also cause an increase in emission threshold, but because the emission amplitude is a nonlinear function of the basilar membrane motion, the exact calculation is complicated. A simple model calculation (Mills 1997) suggests the following approximate result.(1) For small or moderate decreases in cochlear amplifier gain, the corresponding increase in emission threshold will be approximately equal to the decrease in cochlear amplifier gain. This was the case previously illustrated (Mills 2003). (2) For cases of severe dysfunction, in which the gain of the cochlear amplifier is reduced to zero or near zero, the total increase in emission threshold is estimated to be about $10 \mathrm{~dB}$ less than the change in cochlear amplifier gain (see Fig. 8 in Mills 1997). This is the case chosen for illustration here. In this case the normal cochlear amplifier gain is assumed to be about 40 $\mathrm{dB}$ at the relevant parameters, and both dysfunctions are assumed to reduce the gain to approximately zero. Considering just the effect of either dysfunction on the cochlear amplifier gain, therefore, there is an increase of $40 \mathrm{~dB}$ in the $\mathrm{ABR}$ threshold and a corresponding increase of $30 \mathrm{~dB}$ in the emission threshold, as shown. See text for other details.

tion from a normal control at the same frequency (dashed line), as well as the mean threshold level determined for the normal control group. As can be seen, the shift in emission threshold in the experimental animal compared with the normal mean was 
$15 \mathrm{~dB}$ at $2.8 \mathrm{kHz}$ and $25 \mathrm{~dB}$ at $15 \mathrm{kHz}$. For comparison, the ABR threshold in this same animal was determined to be $40 \mathrm{~dB}$ SPL at $2.8 \mathrm{kHz}$. Since the average normal $\mathrm{ABR}$ threshold at this frequency was found to be $18 \mathrm{~dB}$ SPL, this implies that at $2.8 \mathrm{kHz}$ there was an increase of $22 \mathrm{~dB}$ in the ABR threshold for this animal compared with the normal mean.

Measurements such as those in Figure 1 were used to construct threshold-frequency functions for each animal. Examples of cases covering the observed range of dysfunction are presented in Figure 2. Each panel represents threshold data in a form similar to an audiogram. The horizontal axis in each panel is the stimulus frequency on a logarithmic scale. The vertical axis is the difference of the measured threshold from the normal mean. As customary in audiograms, the normal response is defined by the zero level indicated near the top of each panel, and increasing threshold (worse hearing) is plotted downward.

The audiograms shown in Figure 2 span the range of the responses seen in the experimental group. The left column (A) presents a representative animal showing little or no loss. There appears to be the beginning of loss at each frequency extreme but otherwise normal thresholds are seen. Figure 2B is a representative case of those with mild to moderate hearing loss. This animal shows near-normal response in the midfrequencies, slightly more loss at low frequencies, and a pronounced sloping high-frequency loss. In the ABR threshold shift, this loss exceeds 40 $\mathrm{dB}$ at $28 \mathrm{kHz}$. The emission threshold shift is generally somewhat less than the ABR threshold shift measured at the same frequency. This relationship is quantitatively examined in the threshold-shift diagrams in Figures 3-6.

Figure 2C represents the threshold measures for the same animal as in Figure 1 and illustrates (vertical arrows) the threshold-shift calculations discussed there. This animal represents an intermediate case, where the emission shifts are still generally moderate but the ABR threshold increases are more severe. In the ABR for this animal, the high-frequency sloping loss now extends down to about $2 \mathrm{kHz}$, and this progression apparently has eliminated the relatively healthy midfrequency region seen in Figure 2B. The loss at low frequencies is $20-30 \mathrm{~dB}$, increasing to nearly $50 \mathrm{~dB}$ at high frequencies. The ABR response at the highest frequencies is potentially due to cross talk, so the threshold elevations measured at 21 and $28 \mathrm{kHz}$ must be taken as lower limits. As in the other animals, the emission threshold shift is generally parallel to the ABR shift but is less severe.

Figure 2D represents one of the more severe functional losses seen in the study. At low frequencies, up to $3 \mathrm{kHz}$, the $\mathrm{ABR}$ threshold is uniformly elevated about $30 \mathrm{~dB}$. Above this frequency, the elevation increases rapidly. The elevation is at least 60 $\mathrm{dB}$ at $8 \mathrm{kHz}$ and above. As noted, because of possible cross talk, this is actually a lower limit to the ABR threshold elevation at higher frequencies. Again, at all frequencies the emission threshold elevation is typically less than that of the ABR. Even in this severe case, emission thresholds remain below estimated instrumental distortion limits.

As suggested by Figure 2, there is usually greater increase in the ABR threshold than in either of the two emission threshold measures at the corresponding stimulus frequency. A direct method of comparing the threshold shifts in ABR to those in emissions is a threshold-shift plot (Mills 2003). In Figure 3, the change in emission threshold is plotted on the vertical axis against the change in ABR threshold on the horizontal axis. Each point represents one frequency, with different animals represented by different symbols. The same four animals are presented as in Figure 2. The bold arrow suggests the general trend of the relationship as auditory loss goes from mild to severe.

Responses for all the frequencies tested in all animals in the experimental group are summarized in Figure 4. The bold unfilled circles represent all data points that are not limited by ABR cross talk. The right-facing triangles indicate the remaining points in which the ABR threshold measured is actually a lower limit. As suggested by the symbol chosen, the actual values could lie at or to the right of the symbol shown. Regression statistics were calculated using only the certain points (circles). The regression line is shown in both panels as the heaviest solid line. In both panels, the linear regression accounts for about $60 \%$ of the variance, starts near the origin, and increases at a slope of about 0.55 . That is, an increase of $60 \mathrm{~dB}$ in the ABR threshold on average would be associated with an increase of about $33 \mathrm{~dB}$ in the emission threshold. The parallel lighter solid lines indicate the 95\% prediction intervals. As can be seen, the responses in such threshold-shift plots are found to be relatively well localized, particularly for emission parameters $L_{1}=L_{2}+20 \mathrm{~dB}$.

\section{DISCUSSION}

\section{Comparison to previous results}

The regression line found previously for the acute furosemide case (Mills 2003) is shown by a dashed line in each panel of Figure 4 . The regression relationship for the chronic furosemide case considered here is found to be slightly steeper than for the acute case. That is, for the same emission threshold shift, the ABR threshold increase was slightly less when 
furosemide was applied chronically than when it was applied acutely. In other words, at a given emission threshold shift there appears to be a small improvement in ABR threshold in the chronic case compared with the acute response, as expected (see Introduction).

In Figure 5, the results from Figure 4 are compared with those in the previous study of permanent threshold changes due to acoustic damage (Mills 2003). This comparison is particularly relevant because both of these induced conditions mimic naturally occurring pathologies and both are chronic conditions in that natural adaptive responses have been given time to occur. Compared with equal-level stimuli, emissions with unequal-level stimuli clearly provide better separation between the sound damage and low-EP responses. In particular, consider cases in which the ABR shift is more than $20 \mathrm{~dB}$, that is, when at that frequency there is a significant functional loss (to the right of the vertical dashed line, lower panel). There is nearly a complete separation of the responses to the two dysfunctions in this region. In fact, the same line found previously with acute furosemide appears to be adequate in defining the boundary between the two conditions for ABR shift greater than $20 \mathrm{~dB}$. This is the bold dotted line given by the equation (Mills 2003),

$$
\begin{gathered}
\Delta \mathrm{CDT}=0.6 \Delta \mathrm{ABR}+8 \mathrm{~dB}, \text { or, equivalently } \\
(\Delta \mathrm{CDT}-20)=0.6(\Delta \mathrm{ABR}-20) .
\end{gathered}
$$

This boundary line starts at emission and ABR threshold shifts both equal to $+20 \mathrm{~dB}$ and proceeds upward from there with a slope of 0.6. From this equation, for example, the boundary line for an ABR shift of $60 \mathrm{~dB}$ would be located at an emission threshold shift of $44 \mathrm{~dB}$.

\section{Possible cochlear dynamics underlying observed threshold shifts}

The distributions of threshold shifts in Figure 5 also carry potential implications for the possible dynamics of the two dysfunctions considered. Note first that the regression lines for the low-EP condition do pass through the origin but for the acoustic damage condition they do not. This appears to be due to the fact that acoustic exposure in some animals causes an increase in emission threshold without a substantial increase in ABR threshold. Such cases result in the points seen in the region labeled "pre-clinical acoustic damage" in Figure 5. As this label suggests, in humans there is a similar relative weakness in emissions observed in ears with apparently normal hearing when there is significant audiometric threshold elevation in the contralateral ear due to presumptive noise damage (Lucertini et al. 2002). In contrast, note that the low-EP condition does not result in points in the same region (Figs. 4 and 5B).

The fact that the regression lines in chronic low-EP dysfunction tend to go through the origin implies that any EP decrease large enough to cause significant dysfunction appears to simultaneously increase both ABR and emission thresholds. However, these do not generally increase at the same rate. The increase in emission threshold is typically only $56 \%$ of the increase in ABR threshold, i.e., the slope of the regression line in Figure $4 \mathrm{~B}$ is 0.56 . In comparison, the slope of the regression line for the acoustic damage data is 0.77 in the corresponding Figure 5B.

The differences in the observed threshold-shift distributions corresponding to the two underlying conditions can be explained using the following procedure (Mills 2003). In cases where there is substantial auditory impairment, either of the underlying conditions considered here can safely be presumed to cause some dysfunction in the cochlear amplifier. One can then estimate the expected response to be seen in a threshold-shift diagram by considering in sequence: (1) changes due to the decrease in cochlear amplifier gain, taken by itself, and (2) adding changes resulting from all other aspects of the dysfunction. Now, consider two such cases chosen, one of low EP, the other of acoustic damage, so that they have the same decrease in cochlear amplifier gain, at least at some frequency. This means that both dysfunctions can be represented by the same vector $\mathbf{A}$ in Figure 6 , which is the change in emission and ABR thresholds due solely to the change in gain of the cochlear amplifier.

Now the additional contributions of each different dysfunction can be added to the vector $\mathbf{A}$. Consider acoustic damage first. Assuming (1) that acoustic damage operates mainly through the loss of function of OHCs, and (2) that the main effect of OHCs upon the inner hair cells (IHCs) is through the cochlear amplifier, then the additional result of $\mathrm{OHC}$ damage is to decrease the emission amplitude due to the loss of some of their generators, i.e., a decrease in the number of functional OHCs (Trautwein et al. 1996). For pure OHC damage, therefore, there is an additional increase in the emission threshold as indicated by the vertical arrow. Of course, acoustic damage can also cause damage to IHC stereocilia (Liberman and Dobbs 1984). In the case that there is sufficient IHC damage to be directly responsible for an additional ABR threshold shift, the vertical arrow in Figure 6 would tilt to the right (not shown for simplicity).

For the low-EP condition, in contrast, it can be argued that the main additional effect of the low EP, that is otherwise not accounted for or adapted to, is the lessening of the electrical drive to the IHCs. For example, spontaneous eighth nerve firing rates typi- 
cally decline to zero in a low-EP condition, at least acutely (Sewell 1984a, b, c; Rübsamen et al. 1995). The main effect of the lower EP on the OHCs has already been taken into account through the decrease of cochlear amplifier gain. The decreased electrical drive to the IHCs, to the extent that it is not compensated for, can therefore be assumed to cause an additional increase in the ABR threshold with little or no change in the emission threshold (indicated by the horizontal arrow). Additional evidence of the effects of decreased electrical drive to the IHCs with chronic low EP comes from the shallow slopes of the neural input-output functions found both under conditions of chronic furosemide application (Schmiedt et al. 2002a) and in aged gerbils (Mills et al. 1990).

Also note in the threshold-shift plots that the acute low-EP response had a lower slope than the chronic low-EP case (Fig. 4). For the same situation, therefore, the acute response would be expected to lie to the right of the point denoted "Low EP" in Figure 6. This fits with the idea that there are additional effects of abrupt EP decrease on IHC responses over and above those on the OHCs, and that some, but not all, of these effects are resolved or adapted to in the chronic low-EP condition. As noted above, such additional effects on IHC responses are well established in the case of an acute decrease in EP. The idea that some such effects persist in the chronic case is seen to be consistent with the observed threshold-shift responses (Fig. 6).

\section{SUMMARY}

The threshold-shift plot (Fig. 6) demonstrates that observed changes in ABR and emission thresholds are consistent with the hypothesis that the main effect of a chronic low EP is a decline in the cochlear amplifier gain, the "energy starvation" model previously proposed (Schmiedt et al. 2002a). There also appears to be an additional, generally smaller shift in the neural threshold attributable to the lessening of the drive to the IHCs due to the low EP. As Figure 2 illustrates, the cochlear amplifier loss is mostly at high frequencies in mild and moderate cases, at least for furosemide-induced low EP in gerbil. As dysfunction increases, the loss becomes widespread across frequency. Even in severe cases, however, the loss at low frequencies $(<4 \mathrm{kHz})$ is limited to about $30 \mathrm{~dB}$, in contrast to high frequencies where there can be a sloping loss tending to ABR threshold increases exceeding $60 \mathrm{~dB}$. In all cases, the actual decline in cochlear amplifier gain is estimated to be somewhat smaller than the observed ABR shift (Fig. 6).
Comparison of the shifts in emission threshold with those in ABR threshold shows that there is a typical signature associated with chronic EP decrease, where the emission threshold change is typically smaller than the ABR threshold change (Fig. 4). For unequal emission stimulus levels $\left(L_{1}=L_{2}+20 \mathrm{~dB}\right)$, the emission threshold change is about $56 \%$ of the ABR threshold change on average. For acoustic damage, in contrast, at the same ABR threshold increase, the emission threshold increase is typically much larger (Fig. 5). Overall, these results provide additional support for the possibility that simultaneous measurements of emission and neural thresholds can provide a method of differential diagnosis of cochlear dysfunction, as previously demonstrated for the acute low-EP case (Mills 2003).

\section{ACKNOWLEDGMENTS}

Support was provided to DMM by grants DC 04077 and DC 04661 from the National Institute for Deafness and Other Communication Disorders, National Institutes of Health (NIH), and to RAS by a Virginia Merrill Bloedel Traveling Scholar award and by grant AG14748 from the National Institute for Aging, NIH.

\section{REFERENCES}

Felder E, Schrott-Fischer A. Quantitative evaluation of myelinated nerve fibres and hair cells in cochleae of humans with age-related high-tone hearing loss. Hear. Res. 91:19-32, 1995.

Gates GA, Mills DM, Nam B-H, D'Agostino R, Rubel EW. Effects of age on the distortion-product otoacoustic emission growth functions. Hear. Res. 163:53-60, 2002.

Hellstrom LI, Schmiedt RA. Measures of tuning and suppression in single-fiber and whole-nerve responses in young and quietaged gerbils. J. Acoust. Soc. Am. 100:3275-3285, 1996.

Johnsson L-G, Hawkins JE. Strial atrophy in clinical and experimental deafness. Laryngoscope 82:1105-1125, 1972.

LANG H, Schulte BA, Schmiedt RA. Effects of chronic furosemide treatment and age on cell division in the adult gerbil inner ear. J. Assoc. Res. Otolaryngol. 4:164-175, 2003.

Liberman MC, Dodds LW. Single-neuron labeling and chronic cochlear pathology. III. Stereocilia damage and alterations of threshold tuning curves. Hear. Res. 16:55-74, 1984.

Lucertini M, Moleti A, Sisto R. On the detection of early cochlear damage by otoacoustic emission analysis. J. Acoust. Soc. Am. 111:972-978, 2002.

MiLLS DM. Interpretation of distortion product otoacoustic emission measurements. I. Two stimulus tones. J. Acoust. Soc. Am. 102:413-429, 1997.

MiLLS DM. Interpretation of standard distortion product otoacoustic emission measurements in light of the complete parametric response. J. Acoust. Soc. Am. 112:1545-1560, 2002.

MirLs DM. Differential responses to acoustic damage and furosemide in auditory brainstem and otoacoustic emission measures. J. Acoust. Soc. Am. 113:914-924, 2003.

Mills DM, Norton SJ, Rubel EW. Vulnerability and adaptation of distortion product otoacoustic emissions to endocochlear potential variation. J. Acoust. Soc. Am. 94:2108-2122, 1993. 
Mills DM, SHEPHERD RK. Distortion product otoacoustic emission and auditory brainstem responses in the echidna (Tachyglossus aculeatus) J. Assoc. Res. Otolaryngol. 2:130-146, 2001.

Mills JH, Schmiedt RA, Kulish LF. Age-related changes in auditory potentials of Mongolian gerbil. Hear. Res. 46:201-210, 1990.

Pauler M, Schuknecht HF, White JA. Atrophy of the stria vascularis as a cause of sensorineural hearing loss. Laryngoscope 98:754$759,1988$.

Puel J-L, Ruel J, D'Aldin CG, Pujol R. Excitotoxicity and repair of cochlear synapses after noise-trauma-induced hearing loss. Neuroreport 9:2109-2114, 1998.

Rosenhall U, Pederson K, Svanborg A. Presbycusis and noise-induced hearing loss. Ear Hear. 11:257-263, 1990.

Rübsamen R, Mills DM, Rubel EW. Effects of furosemide on distortion product otoacoustic emissions and on neuronal responses in the anteroventral cochlear nucleus. J. Neurophysiol. 74:1628-1638, 1995.

SCHMIEDT RA. Effects of aging on potassium homeostasis and the endocochlear potential in the gerbil cochlea. Hear. Res. 102:125-132, 1996.

Schmiedt RA, Mills JH, Adams JC. Tuning and suppression in auditory nerve fibers of aged gerbils raised in quiet or noise. Hear. Res. 45:221-236, 1990.

Schmiedt RA, Lang H, Okamura H, Schulte BA. Effects of furosemide applied chronically to the round window: A model of metabolic presbycusis. J. Neurosci. 22:9643-9650, 2002a.
Schmiedt RA, Okamura H-O, Lang H, Schulte BA. Ouabain application to the round window of the gerbil cochlea: A model of auditory neuropathy and apoptosis. J. Assoc. Res. Otolaryngol. 3:223-233, 2002b.

SCHUKNECHT HF. Further observations on the pathology of presbycusis. Arch. Otolaryngol. 80:369-382, 1964.

SCHUKNeCht HF, GACEK MR. Cochlear pathology in presbycusis. Ann. Otol. Rhinol. Laryngol. 102(P2 Suppl. 158):1-16, 1993.

Schulte BA, Schmiedt RA. Lateral wall Na,K-ATPase and endocochlear potentials decline with age in quiet-reared gerbils. Hear. Res. 61:35-46, 1992.

SEWELL WF. The effects of furosemide on the endocochlear potential and auditory-nerve fiber tuning curves in cats. Hear. Res. 14:305-314, 1984a.

SEWELL WF. Furosemide selectively reduces one component in ratelevel functions from auditory-nerve fibers. Hear. Res. 15:69-72, 1984b.

SEWELL WF. The relation between the endocochlear potential and spontaneous activity in auditory nerve fibres of the cat. J. Physiol. (London) 347:685-696, 1984c.

Tarnowski BI, Schmiedt RA, Hellstrom Li, Lee FS, Adams JC. Agerelated changes in cochleas of mongolian gerbils. Hear. Res. 54:123-134, 1991.

Trautwein P, Hofstetter P, Wang J, Salvi R, Nostrant A. Selective inner hair cell loss does not alter distortion product otoacoustic emissions Hear. Res. 96:71-82, 1996. 